\title{
Specimen Handling and Tissue Preparation in Pre-analytical Phase of HER2 Testing at Tertiary Referral Hospitals of Metro Manila, Philippines
}

\author{
Marlowe S. Imperial, ${ }^{1}$ Susano B. Tanael, ${ }^{1}$ Jose Ma C. Avila ${ }^{2}$ \\ and Ma. Lourdes A. Tiambeng ${ }^{3}$ \\ ${ }^{1}$ Section of Medical Oncology, Department of Medicine, College of Medicine and Philippine General Hospital, University of the Philippines Manila \\ ${ }^{2}$ Department of Pathology, College of Medicine, University of the Philippines Manila \\ ${ }^{3}$ Department of Medicine, Cardinal Santos Medical Center, Mandaluyong City
}

\begin{abstract}
Introduction. HER2 test result depends on a good quality histological section of the breast specimen. Laboratories that process specimens using sophisticated and molecular testing should receive suitable specimen for more accurate results and less waste of expensive resources.

Methods. A questionnaire based on the ASCO-CAP Guidelines for HER2 testing was developed and administered to pathologists from the local laboratories of Metro Manila-Philippines tertiary referral hospitals. Questionnaire responses were analyzed using descriptive statistics.
\end{abstract}

Results. Most laboratories did quality control/quality assessment monitoring for the pre-analytic $c_{s}$ analytic $c_{s}$ post-analytic and turnaround time phases. Many transported their specimens from the operating room to the laboratory $<6$ hours but many did not record the duration of fixation. $86 \%$ used $10 \%$ neutrallybuffered formalin but only 4 laboratories (57\%) used the prescribed 10:1 volume to volume ratio of the fixative to the tissue specimen.

Conclusion. Even in standardized procedures, small variations in sample processing for IHC and FISH can still occur. Inexperienced laboratories will have greater problems interpreting HER:2 status results. Laboratories should look into their system in handling specimens for an accurate HER2 testing towards quality assurance.

Key Words: specimen handling, tissue preparation, HER2 testing

Corresponding author: Marlowe S. Imperial, MD

Section of Medical Oncology

Department of Medicine

Philippine General Hospital

University of the Philippines Manila

Taft Avenue, Ermita, Manila 1000 Philippines

Tele/Fax: +632 5263775

Email: docmarlowe@gmail.com

\section{Introduction}

The discovery of the human epidermal growth factor receptor-2 (HER2) in breast cancer provided hope for breast cancer patients since around $15-25 \%$ of these patients overexpress this receptor in their tumors. ${ }^{1,2}$ It is both a prognostic factor for an aggressive tumor behavior ${ }^{3,4}$ as well as a predictive factor of response to treatment. ${ }^{4-6}$

HER2 testing is expensive hence strict standardization of the assay is vital to achieve an accurate result, which identifies the patients who will benefit from trastuzumab, a monoclonal antibody against HER2 overexpression. ${ }^{7}$

Currently, there are two ways of doing HER2 testing, through immunohistochemistry (IHC) and fluorescence in situ hybridization (FISH). IHC uses antibodies to detect expression of HER2 protein on the surface of tumor cells. The level of HER2 protein expression is assessed semiquantitatively by the intensity and percentage of staining. Scores assigned are from 0 to $3+$, wherein 0 and $1+$ are considered negative, $2+$ equivocal and $3+$ positive. ${ }^{7}$ FISH is done by determining the HER2 gene copy number and use of DNA probes. The HER2 fluorescent signal is usually expressed as a ratio relative to the signal for the centromere of chromosome 17.7 IHC $3+$ and a ratio of HER2/CEP $17>$ 2.2 indicate that trastuzumab therapy should be initiated. However, an equivocal IHC result $(2+)$ indicates further reflex testing with FISH to know if the patient will benefit from trastuzumab treatment. ${ }^{8}$

Standardization of the process of handling the specimen is very important since current HER2 tests are subject to both analytic and inter-observer variation. ${ }^{7}$ Laboratory error as defined by the International Organization for Standardization (ISO) is any defect from ordering the tests to reporting the results and appropriately interpreting and reacting to these. ${ }^{9}$

Most of the errors happen during the pre-analytic period in poorly standardized processes. ${ }^{10}$ In one ISO study, the pre-analytic error was as high as $85 \% .{ }^{9}$ A significant 7$20 \%$ error may impact patient care. ${ }^{11}$

Further, the ASCO-CAP guidelines claim that $20 \%$ of HER2 assays performed in the real world were incorrect ${ }^{8}$ 
and this may be attributed to the error in handling the specimen.

Quality programs in the analytic and post-analytic phase, quality control and/or quality assurance, can realize significant positive outcomes by attending to the issues of handling and processing tissue/biopsy specimens. The preanalytic phase is a very critical part since it has the power to direct patient management especially in oncology. Medical oncologists rely heavily on the histological reports of a biopsy specimen.

In a country like the Philippines where healthcare is non-reimbursed and quality initiatives for healthcare are far from ideal, major laboratories processing specimen for more sophisticated and molecular testing ought to receive suitable specimens for more accurate results and less waste of expensive resources. Identifiable standards for specimen collection and handling should be promoted among laboratories. These laboratories should also be monitored and assessed for continuous provision of good quality service to Filipino patients.

This study identifies practice patterns in the handling and processing of tissue/biopsy specimens and possible causes of unsuitable specimen for the analytic phase of HER2 testing; it assesses QC/QA practices of the laboratories of seven tertiary referral hospitals within Metro Manila, aiming for a multidisciplinary HER2 testing in the Philippines.

\section{Methods}

Participants of this cross-sectional study were pathologists requested from the local laboratories of tertiary referral hospitals located in Metro Manila-Philippines that have participated in UK NEQAS External Quality Assurance Program - Cardinal Santos Medical Center, Makati Medical Center, National Kidney and Transplant Institute, Philippine General Hospital, Philippine Kidney and Dialysis Foundation, Saint Luke's Medical Center, The Medical City, and University of Santo Tomas. Only seven of these laboratory institutions participated in this study.

A questionnaire on breast specimen handling for HER2 testing was developed based on the ASCO-CAP Guidelines ${ }^{1}$ for HER2 testing, for exploratory and descriptive purposes eliciting information on the sample handling, duration of fixation, type of fixative, laboratory profiles and quality indicators monitored by the laboratory. The questionnaire was given to the pathologist of the laboratory and was retrieved after a week. Identities of the respondent and corresponding laboratory were masked. Questionnaire responses were analyzed for the entire group using descriptive statistics.

\section{Results and Discussion}

\section{Breast Specimen Handling for HER2 Testing}

Most institutions received HER2 testing request for 10$25 \%$ of breast tumor samples, coming mostly from within the same institution (Figure 1). There were 1-9\% of breast tumor samples received from other institutions for HER2 testing. Tumor samples received for HER2 testing were from core needle or excision specimen.

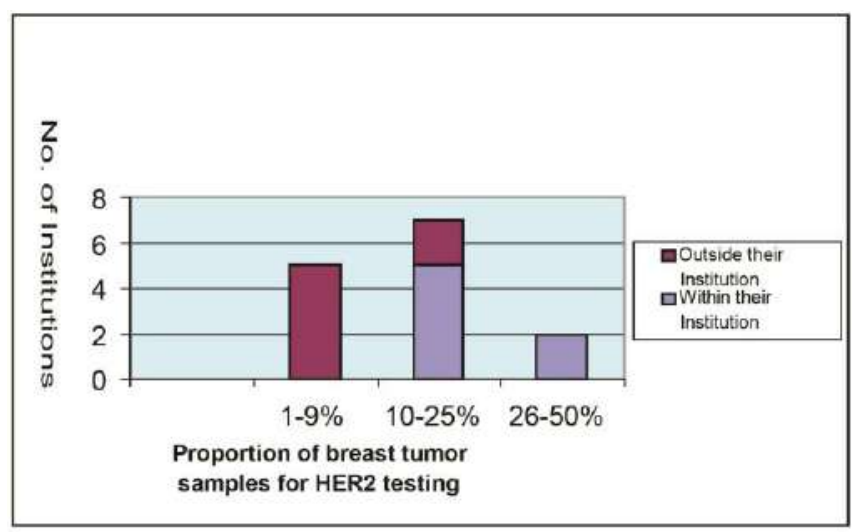

Figure 1. Breast tumor samples for HER2 testing received in a month.

Adequate preparation of breast tissue specimen for evaluation should be properly observed for an accurate histologic diagnosis and HER2 testing. Time of tissue acquisition to tissue fixation should be as short as possible. . $^{, 12}$ However, there is no prescribed duration.

Four $(57 \%)$ stated that their institutions took around $>1$ hour but $<6$ hours to transport the specimen from the operating room to the laboratory and 1 said it was $>6$ hours but $<12$ hours. There were 2 who said $>30$ minutes but $<1$ hour. Specimens should be transported ideally in the fresh state, oriented and inked. Delayed fixation will result in loss of mitotic figures that may result to downgrading of invasive carcinomas. HER2 proteins are also fixation sensitive, and poor fixation can result in IHC false-negative results. ${ }^{12}$ Many knew that breast specimen after excision or biopsy should be collected, placed in a specific area in the operating room and transported to the pathology laboratory as a batch. The differences in time of transport to the laboratory probably lie on the waiting period of transporting the specimens as a batch.

All received their specimen with formalin. However, the volumes of the formalin fixative to tissue ratio were varied across the different laboratories. Quality of the sections made from the tissue depends upon adequate fixation; the fixative should be $10 x$ the volume of the specimen. ${ }^{13}$ The 
fixative should be $10 \%$ neutral buffered formalin. ${ }^{8}$ Many laboratories (86\%) used 10\% neutral-buffered formalin. However, the volume to volume ratio of fixative to sample excised specimen varied from $<1: 1$ to $1: 1$, to $10: 1$ to $>10: 1$. Only 4 laboratories (57\%) used the 10:1 volume to volume ratio of the fixative to the tissue specimen. This was better compared to the study by M Sharif ${ }^{13}$ which was only $35 \%$. The duration from excision to fixation and the duration of fixation process were not recorded nor monitored by many (Table 1). This is not in accordance with the ASCO-CAP consensus guidelines, which is at least 6 hours to not more than 48 hours. ${ }^{8}$

The breast tissue specimen should be serially sliced at 3$5 \mathrm{~mm}$ intervals. ${ }^{12}$ Around 5 laboratories sliced the breast tissue specimen $<5 \mathrm{~mm}$ interval. Only 2 sliced their specimen with a $5-10 \mathrm{~mm}$ interval.

Proper patient identification by using unique accession numbers was being observed by all laboratories including usage of disposable blades and having a system for checking expired chemicals (Table 1). Only 57\% of the laboratories recorded the number of tissues passed through the chemicals everyday and each of them had its own predetermined limit of tissue pass for compulsory change of chemicals (e.g., once the container was full, every 1000 cassettes, every 15000 blocks, or every 7 days). Microwave was not employed as a heating method for antigen retrieval nor further sample processing.

Table 1. Laboratory practices in handling breast tissue specimen.

\begin{tabular}{lc}
\hline \multicolumn{1}{c}{ Laboratory Practices } & No. of Respondents \\
\hline $\begin{array}{l}\text { Use microwave for quick sample processing } \\
\text { Use paraffinized block sections for HER 2 testing }\end{array}$ & 0 \\
if cut six weeks earlier & 0 \\
$\begin{array}{l}\text { Request fixation time from excision to start of } \\
\text { fixation }\end{array}$ & 2 \\
$\begin{array}{l}\text { Request record of duration of fixation } \\
\text { Use a unique accession number for correct patient }\end{array}$ & 1 \\
$\begin{array}{l}\text { identification } \\
\text { Record the number of tissues passed through the } \\
\text { chemicals every day }\end{array}$ & 7 \\
$\begin{array}{l}\text { Have a predetermined limit of tissue pass for } \\
\text { compulsory change of chemicals }\end{array}$ & 4 \\
$\begin{array}{l}\text { Have a system of checking for expired chemicals } \\
\text { Knife blades are disposable }\end{array}$ & 4 \\
\hline
\end{tabular}

Proper control of the pre-analytical process involved having standard operating procedures for sample accession, identification, acceptance or rejection. Examination of gross specimen and sampling should also be observed and documented. There must also be a system for regular changing of chemicals used for processing based on the number of tissues passed through. The use of controls, calibrated microtome and disposable blades must be practiced all of the time. ${ }^{14}$

Five laboratories had a system of monitoring the preanalytic parameters (Table 2). This finding was better than the study by Mohammad wherein 34\% of the clinical details were missed. ${ }^{13}$

Table 2. Pre-analytic parameters monitored

\begin{tabular}{lc}
\hline \multicolumn{1}{c}{ Parameters Monitored } & No. of Respondents \\
\hline Erroneous tissue label & 5 \\
Erroneous laterality of breast tissue & 5 \\
Missing patient name & 4 \\
Missing physician identification & 4 \\
Specimen lost & 4 \\
Missing clinical diagnosis & 3 \\
\hline
\end{tabular}

\section{Laboratory Profile}

All laboratories received a huge amount of specimen for analysis. There were 3 that had an average of 1,000 specimen annual load for their Surgical Pathology Service. The other 3 received $>300,800$ and 1,500 specimens, respectively. For the Anatomic Pathology service, only 3 revealed their profile; each stated 2,000, 3,400 and 5,000 specimen load every year.

ER/PR testing was done by 5 laboratories; 2 had $<50$ average specimen load in a month. Another 2 processed around $>80$ specimen and the remaining had an average of 50-80 specimen load every month.

IHC HER2 testing was done in 5 laboratories; 2 received $<40$ specimens in a month while another 2 had $>80$ specimens on the average; 1 examined 50-80 specimens per month. The laboratories conformed to the recommendation by the national consensus in $\mathrm{UK}^{15}$ where it is suggested that laboratories undertaking a minimum of 250 tests per year should provide IHC HER2 testing services. ${ }^{15}$ Of these 5 laboratories, there were usually 5 to 14 pathologists who read IHC testing for HER2. Dako commercial kit for IHC testing was employed by 3 laboratories while the other two used Invitrogen kit.

Majority (5) did not process FISH HER2 testing. Of the 2 who were capable of doing FISH testing, around 5-20 specimens per month were being tested and this was in compliance with the recommendation of the national consensus in $\mathrm{UK}^{15}$ (100-150 FISH testing in a year). PathVision test was the FISH HER2 commercial kit used.

Parallel testing is done to validate one's result. Validation of a test must be done before it is offered. Test validation requires 25-100 samples tested by alternative validated method. Concordance rate should be $95 \%$ in testing negative and positive HER2 categories. ${ }^{8}$

Five laboratories practiced parallel testing. Each had different minimum number of cases/known HER2 slides used. Most had at least 1 slide used as a control. Only one reported having 50 HER2 slides which was in accordance with the ASCO-CAP consensus guideline ${ }^{15}$. Four laboratories had 1-3 pathologists to interpret validation results. One reported having 4-10 pathologists. Of these, only 3 reported their average concordance rate, where 2 gave a $90-99 \%$ concordance rate while the remaining had $100 \%$ concordance rate. This was relatively higher than that 
reported in one study where $\mathrm{k}$ statistics was only 0.67 and 0.74. ${ }^{16}$ However, majority did not have a reference laboratory to perform parallel testing. Accrediting authorities insist that every laboratory should have some form of external quality assessment for all the tests performed under its scope of activity. ${ }^{17}$

A positive HER2 test is defined as either IHC $3+$ cell surface protein expression or FISH result of amplified HER2 gene copy number (average of $>6$ gene copies/nucleus) or HER2/CEP 17 ratio of >2.2.8,16,18 All laboratories reported that they use an IHC $3+$ amplified as their positive control material; 6 had controls available in several blocks. Of these, 3 laboratories changed the blocks monthly from which the controls were obtained; 2 depended on when the samples became available while the remaining institution changed their blocks every batch. All laboratories did not use control cell lines preparation for HER2 testing.

There were 4 laboratories that used negative internal control and each used different negative internal control. Some of the negative controls used were neuroepithelial/stromal cells and an IHC 0/1.

Six laboratories repeated the assay if controls did not show the expected results in an HER2 testing batch. Only one rejected the assay and repeated the testing.

Table 3 revealed that many laboratories had an ongoing competency assessment for HER2 testing. All were particular about troubleshooting for staining errors and staining interpretation. Many also monitored their competency in adequate tissue recognition, inventory control and scoring.

Table 3. Competency Assessment of Laboratories capable of HER2 testing.

\begin{tabular}{lc}
\hline Competency Assessment & Number of Respondents \\
\hline Troubleshooting for staining errors & 5 \\
Staining Interpretation & 5 \\
Adequate Tissue Recognition & 4 \\
Inventory Control & 4 \\
Scoring & 4 \\
\hline
\end{tabular}

Five laboratories (71\%) had no standardized criteria for detection of unsuitable specimen. For the two who had standard criteria of rejecting unsuitable specimen, they only usually reject the specimen. However, according to the recommendations published in $2007,,^{10}$ it is very important to identify the reason/s why the specimen is unsuitable and give recommendations. It is also important to notify the attending physician and communicate the findings and recommendations.

\section{Quality Control/Quality Assurance Profile}

Pre-analytical phase includes providing appropriate clinical history, proper patient preparation, proper collection of specimen, proper preparation of samples and assurance that the testing equipment is accurate and precise in testing. ${ }^{8,11,19,20}$ Most of the elements in the pre-analytical phase aim to improve the accuracy of the diagnosis. ${ }^{20}$

All pre-analytic indicators were monitored by the 7 laboratories (Figure 2). All were particular on the specimen identification and accessioning errors. Of these, specimen or patient identification is the most important. ${ }^{20}$ However, 5 laboratories did not assess the adequacy of the clinical history. This finding was also similar with the study of $\mathrm{M}$ Sharif ${ }^{13}$ wherein $34 \%$ had inadequate clinical history. It is important to educate health professionals through manuals and interdepartmental meetings on how to properly collect, handle and prepare the specimen because it greatly impacts management. ${ }^{10}$

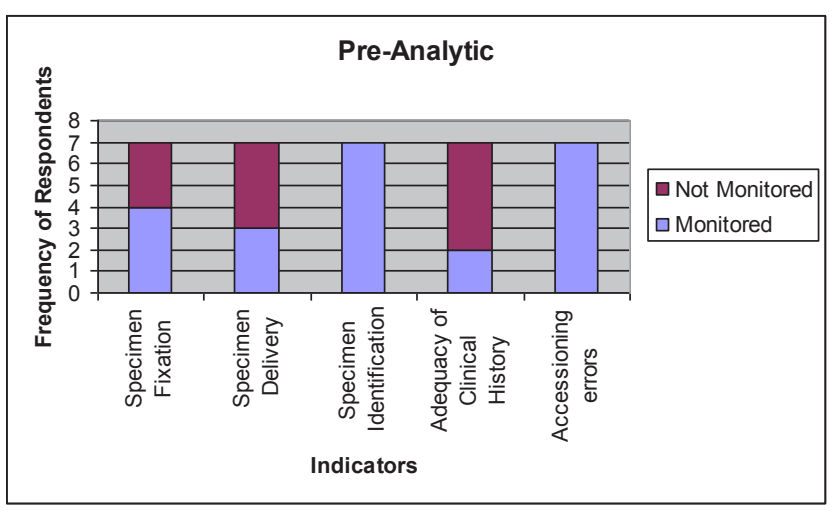

Figure 2. Pre-analytic Indicator Laboratory Profiles

Analytical phase begins with gross examination of the specimen and ends with diagnosis. Most important in this phase is the provision of the diagnosis. ${ }^{20}$ Its indicators include actual test performance and result calculation, and availability of add-on testing and repeat testing. Participation in external reviews of analytic testing is also included. $8,11,19$

Block labeling and slide labeling errors topped the analytic indicators monitored by all laboratories (Table 4). Six laboratories checked the frozen section-permanent section concordance, slide quality; 5 checked final diagnosis, histology and gross room monitor. There was a poor monitoring noted for the annual inventory of antibodies and frequency of use and its external validation. The latter may be because many institutions did not have a reference laboratory for external quality assessment.

The post-analytic indicators were adequately monitored by all laboratories specifically the transcription and verification errors (Figure 3). Post-analytical phase includes routine generation and transmission of test results, and billing issues. $8,11,14,20$ As high as 16\% laboratory mistakes are due to the post-analytical error. Majority of these is from failing to correct an erroneous result and not notifying the physician. ${ }^{11}$ 
Table 4. Analytic Indicator Laboratory Profiles

\begin{tabular}{lcc}
\hline \multicolumn{1}{c}{ Analytic Indicators } & Monitored & Not Monitored \\
\hline Block labeling errors & 7 & 0 \\
Slide labeling errors & 7 & 0 \\
Frozen Section- permanent section & 6 & 1 \\
concordance & & \\
Slide quality & 6 & 1 \\
Final Diagnosis & 5 & 2 \\
Histology and gross room monitor & 5 & 2 \\
Peer review error rate & 4 & 3 \\
Immuno-histochemistry & 4 & 3 \\
Intra-Operative & 3 & 4 \\
Frequency of repeat slides & 3 & 4 \\
Annual inventory of antibodies and & 2 & 7 \\
frequency of use & & \\
External validation of selected & 0 & \\
antibodies & & \\
\hline
\end{tabular}

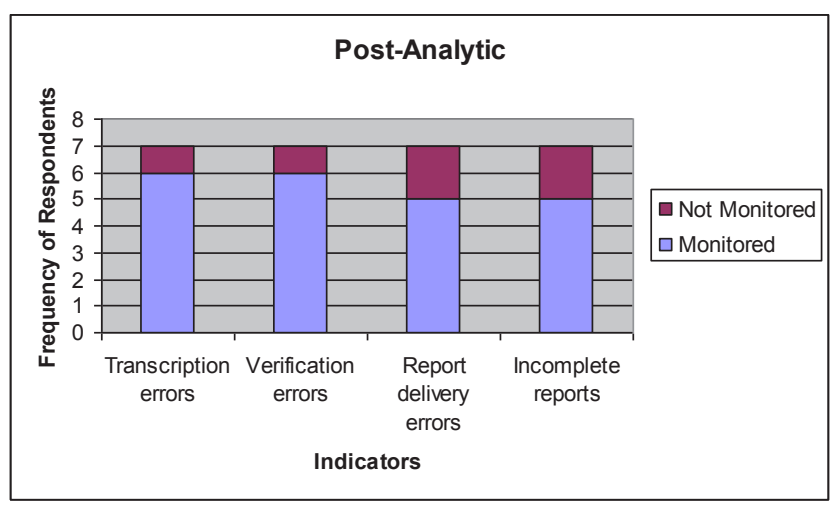

Figure 3. Post-Analytic Indicator Laboratory Profiles

Turnaround time was also checked by all especially the frozen section and biopsy (Figure 4). However, only 3 laboratories monitored for the preliminary and final necropsy results. Timely reporting of interpretive result is very important in patient care because a delay of this may result to delayed management. ${ }^{11}$ Laboratories should strive hard to sign out majority of the cases with 48 hours of receipt of the specimen. ${ }^{14}$

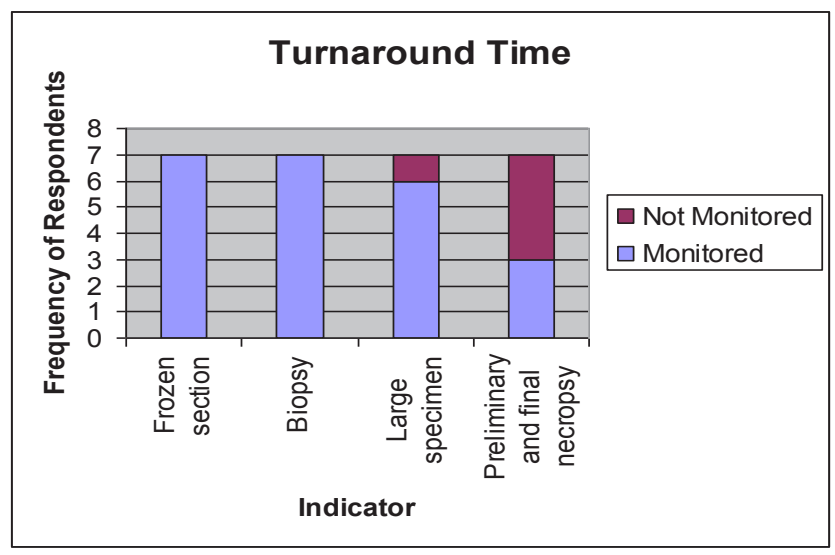

Figure 4. Turn-around Time Indicators Laboratory Profiles
According to Nakleh ${ }^{20}$, the customer or clinician's satisfaction is one of the most vital measures of quality because it gives an insight into the clinician's perception of the laboratory. However, majority of the laboratories had poor monitoring of the clinician satisfaction indicators (Figure 5). Most laboratories only assessed their quality up to the turnaround time.

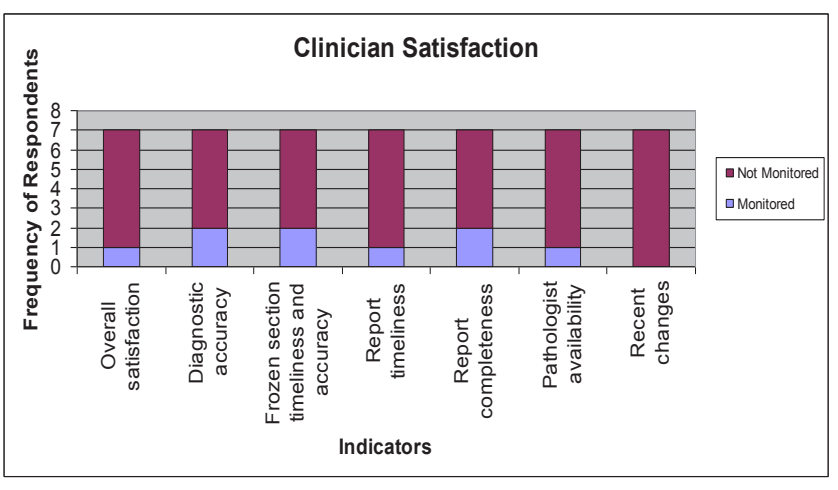

Figure 5. Clinician Satisfaction Indicators Laboratory Profiles

\section{Summary}

The laboratories are receiving a large amount of breast specimens to be tested, hence there is a high demand for HER2 testing.

Even in standardized procedures, small variations in sample processing for IHC and FISH can still occur; the relative experience of laboratory personnel could potentially influence the results obtained by different laboratories. It is expected that inexperienced laboratories will have greater problems interpreting HER2 status results.

Handling of breast tissue specimen is a very critical step needing a very organized and standardized approach. Laboratories should look upon their system in handling of the specimen for an accurate HER2 testing. Major areas to be revisited are the recording of the duration of time from excision to fixing the specimen and the duration of fixing. The process of fixing the specimen should also be properly observed from the use of the fixative solution to the volume used in the fixation process. Laboratories should ensure proper identification of the specimen and provide the patient an accurate histologic diagnosis by observing proper handling of specimens. Laboratories should also monitor clinician's satisfaction of their histologic report.

\section{References}

1. Hynes NE, Stern DF. The biology of erbB-2/neu/HER-2 and its role in cancer. Biochim Biophys Acta. 1994; 1198(2-3):165-84.

2. Owens MA, Horten BC, Da Silva MM. HER2 Amplification ratios by flourescence in situ hybridization and correlation with immunohistochemistry in a cohort of 6556 breast cancer tissues. Clin Breast Cancer. 2004; 5(1):63-9. 
3. Press MF, Bernstein L, Thomas PA, et al. HER-2/neu gene amplification characterized by fluorescence in situ hybridization: poor prognosis in node-negative breast carcinomas. J Clin Oncol. 1997; 15(8):2894-904.

4. Perez EA. Her-2 as a prognostic, predictive, and therapeutic target in breast cancer. Cancer Control. 1999; 6(3):233-40.

5. Paik S, Park C. HER-2 and choice of adjuvant chemotherapy in breast cancer. Semin Oncol. 2001; 28(4):332-5.

6. Slamon DJ, Leyland-Jones B, Shak S, et al. Use of chemotherapy plus a monoclonal antibody against HER2 for metastatic breast cancer that overexpresses HER-2. N Engl J Med. 2001; 344(11):783-92.

7. Dowsett M, Hanna WM, Kockx M, et al. Standardization of HER2 testing: results of an international proficiency-testing ring study. Mod Pathol. 2007; 20(5):584-91.

8. Wolff AC, Hammond ME, Schwartz JN, et al. American Society of Clinical Oncology/College of American Pathologists guideline recommendations for human epidermal growth factor receptor 2 testing in breast cancer. J Clin Oncol. 2007; 25(1):118-45.

9. Wiwanitkit $\mathrm{V}$. Types and frequency of pre-analytical mistakes in the first Thai ISO 9002:1994 certified clinical laboratory, a 6-month monitoring. BMC Clin Path. 2001; 1(1):5.

10. Lippi G, Banfi G, Buttarello M, et al. Recommendations for detection and management of unsuitable samples in clinical laboratories. Clin Chem Lab Med. 2007; 45(6):728-36.

11. Hollensead SC, Lockwood WB, Elin RJ. Errors in pathology and laboratory medicine: consequences and prevention. J Surg Oncol. 2004; 88(33):161-81.
12. Provenzano E, Pinder SE. Guidelines for the handling of benign and malignant surgical breast specimens. Current Diagnostic Pathology. 2007; 13(2):96-105.

13. Sharif MA, Mushtaq S, Mamoon N, Jamal S, Luqman M. Clinician's responsibility in pre-analytical quality assurance in histopathology. Pak J Med Sci. 2007; 23:720-3.

14. Iyengar JN. Quality Control in the histopathology laboratory: An overview with stress on the need for a structured national external quality assessment scheme. Indian J Pathol Microbiol. 2009; 52:1-5.

15. Dowsett, M, Hanby AM, Laing R, et al. HER2 Testing in the UK consensus from a national consultation. J Clin Pathol. 2007; 60(6):685-9.

16. Sauter G, Lee J, Bartlett JM, Slamon DJ, Press MF. Guidelines for human epidermal growth factor receptor 2 testing: biologic and methodologic considerations. J Clin Oncol. 2009; 27(8):1323-33.

17. Assuring quality of examination procedures. Clause 5.6. In International Standard IS/ISO 15189. Medical Laboratories - Particular requirements for quality and competence. 2nd ed. ISO; 2007, p. 22-3.

18. Bartlett JM, Going JJ, Mallon EA, et al. Evaluating HER2 amplification and overexpression in breast cancer. J Pathol. 2001; 195(4):422-8.

19. Wiley EL, Diaz LK. High-quality HER-2 testing: setting a standard for oncologic biomarker assessment. JAMA. 2004; 291(16):2019-20.

20. Nakhleh RE. What is quality in surgical pathology? J Clin Pathol. 2006 59(7):669-72.

\section{Acta Medica Pfilippina \\ THE NaTional Health SCIENCE JOURNAE

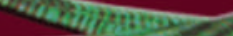 Pubfishing since 1939}

\section{Acta is now accepting membership}

Privileges of members:

- Allows you to submit articles for possible publication

- Have access to all the articles in the website (archives included) which can be downloaded and printed in pdf format

- Advertise your products/services in the available spaces of the website (for approval of the Editor-in-chief)

For details, please visit our website at www. actamedicaphilippina.com.ph or e-mail us at businessmanager@actamedicaphilippina.com.ph for any questions or queries. 